\title{
A Rare Case of Pediatric Cortical Aneurysm Rupture in an Eight-Year-Old Boy
}

\author{
Anooja Abdul Salam ${ }^{1}$ Imogen Ibbett ${ }^{2}$ Nova Thani ${ }^{3}$ \\ 1Division of Medicine and Surgery, Department of Public Health, \\ Royal Hobart Hospital, Affiliated to University of Tasmania, Hobart, \\ Tasmania, Australia \\ 2Department of Medicine and Surgery, Mater Hospital Brisbane, \\ South Brisbane, Australia \\ ${ }^{3}$ Department of Neurosurgery, Royal Hobart Hospital, Affiliated to \\ University of Tasmania, Hobart, Tasmania, Australia \\ Indian J Neurosurg 2022;11:168-170
}

\begin{abstract}
Address for correspondence Anooja Abdul Salam, MBBS, Division of Medicine and Surgery, Department of Public Health, Royal Hobart Hospital, Affiliated to University of Tasmania, 48 Liverpool Street, Hobart, Tasmania 7000, Australia (e-mail: abcanu@gmail.com).
\end{abstract}

\author{
Abstract \\ Keywords \\ - acute intracerebral \\ hemorrhage \\ - cortical cerebral \\ aneurysm \\ - pediatric
}

Aneurysmal subarachnoid hemorrhage in the pediatric population is an extremely rare condition. We present an acute intracerebral hemorrhage in an 8-year-old boy, secondary to a ruptured middle cerebral artery cortical branch (M4 segment) aneurysm. There have been very few cases of cortical aneurysm reported in adults and we did not identify any pediatric cases in the literature to date.

\section{Case Presentation}

An 8-year-old boy was found collapsed in the bathroom at home. On arrival to a regional hospital, he had a Glasgow Coma Score of 3 and was intubated.

His computed tomography (CT) brain and angiogram (-Figs. 1 and 2) showed large frontoparietal intraparenchymal hemorrhage measuring $61 \times 39 \times 48 \mathrm{~mm}$, with a $14 \mathrm{~mm}$ spherical hyperdense lesion within the hematoma. Upon contrast administration, this lesion avidly enhanced, suggesting an arterial filling body-likely an aneurysm. There was significant mass effect with $4 \mathrm{~mm}$ subfalcine herniation to the right. He was transferred to a tertiary care center via air ambulance approximately within 2 hours, during which he developed a unilateral fixed dilated left pupil.

\section{Background Medical History}

Severe autism, attention deficit hyperactive disorder, and behavioral exacerbations with explosive violent outbursts are the background medical history.

published online May 28, 2021
DOI https://doi.org/ $10.1055 / \mathrm{s}-0041-1731251$ ISSN 2277-954X.

\section{Operation}

Upon arrival at the tertiary center, he immediately underwent emergency craniotomy. Intraoperatively, the hemorrhage was evacuated. An aneurysmal mass was noted to arise from the cortical artery over the parietal lobe with clear evidence of recent rupture. Due to the relative size of the aneurysm $(\sim 15 \mathrm{~mm})$ and the parent vessel (approximately $<2 \mathrm{~mm})$, the aneurysm had a fusiform configuration despite being spherical in shape. The distal branch territory was damaged by the hematoma. Hence, the aneurysm was obliterated by clipping the proximal artery. The aneurysm was resected for histopathological analysis. An ipsilateral external ventricular drain (EVD) was placed for monitoring and cerebrospinal fluid (CSF) drainage.

During the following 6 hours, the patient developed raised intracranial pressures (ICP). Repeat CT showed trapping of the contralateral ventricle due to persistent subfalcine herniation. A contralateral EVD was inserted for CSF diversion. Over the next 48 hours, he had a persistently raised ICP despite maximal medical management and aggressive CSF drainage. A further CT scan demonstrated hypodensity in the basal ganglia; retrospectively, this was evolving

(c) 2021. Neurological Surgeons' Society of India.

This is an open access article published by Thieme under the terms of the Creative Commons Attribution-NonDerivative-NonCommercial-License, permitting copying and reproduction so long as the original work is given appropriate credit. Contents may not be used for commercial purposes, or adapted, remixed, transformed or built upon. (https://creativecommons.org/licenses/by-nc-nd/4.0/)

Thieme Medical and Scientific Publishers Pvt. Ltd. A-12, 2nd Floor, Sector 2, Noida-201301 UP, India 

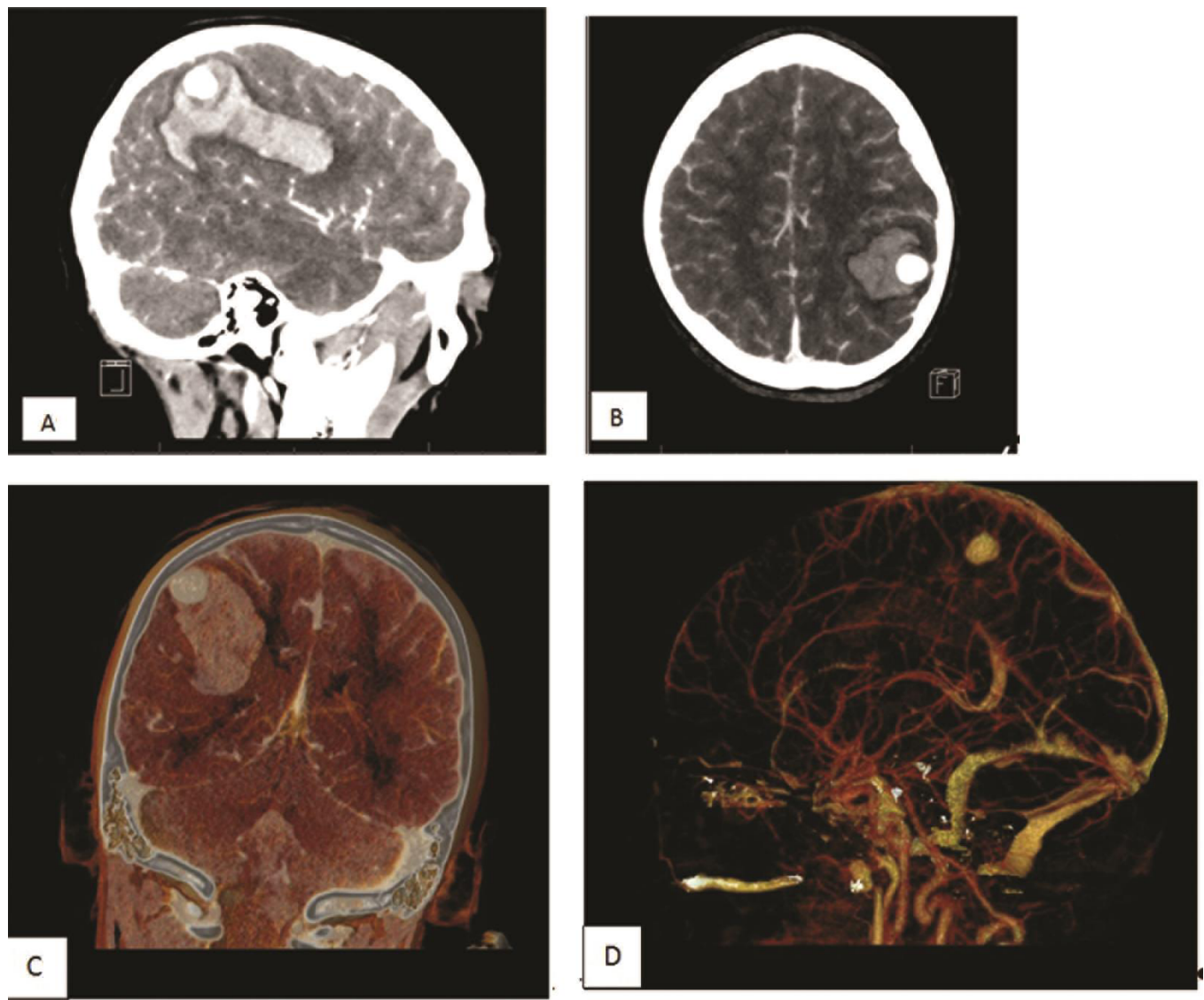

Fig. 1 Cranial computed tomography (CT) scan. (A) Sagittal and (B) axial showing large frontoparietal intraparenchymal hemorrhage with uniformly enhancing lesion. (C) Coronal and (D) sagittal images with three-dimensional reconstruction of CT scan and CT angiogram demonstrating cortical aneurysm.
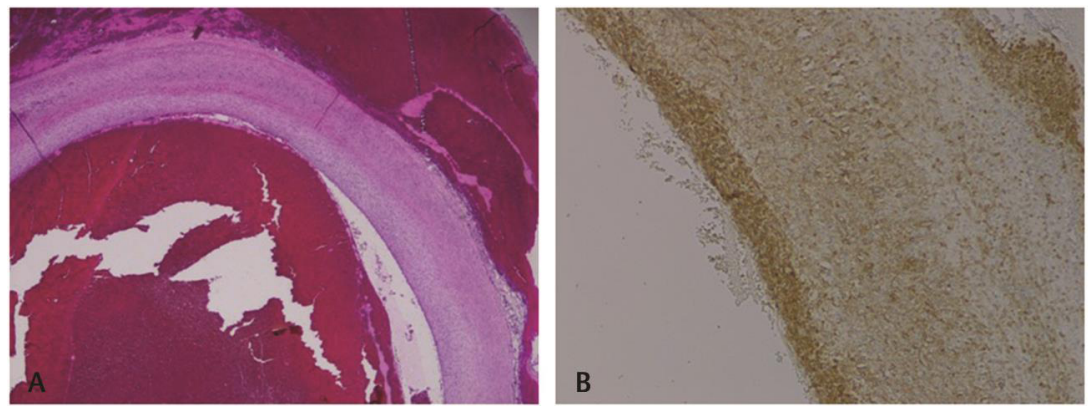

Fig. 2 (A) Hematoxylin and eosin stain of the aneurysm. (B) Immunohistochemical stain.

on previous scans. Although hypoxic injury was suspected, given ongoing intracranial hypertension, decompressive craniectomy was performed, whereupon ICP stabilized. All the investigations were negative for infective etiology.

Magnetic resonance imaging scan of the brain, performed on the seventh postoperative day, demonstrated extensive hypoxic brain injury. It was thought that this was most likely sustained during initial collapse. The patient developed generalized tonic-clonic seizures and showed minimal evidence of neurological improvement. He was palliated and subsequently died.

\section{Histopathology Report}

Histopathology specimen was suggestive of a ruptured vessel with an attenuated wall in keeping with an aneurysm ( - Fig. 2A). The immunohistochemical stain positive for CD31 (brown staining) confirmed that the structure is a vessel (-Fig. 2). 


\section{Discussion and Review of Literature}

Pediatric intracranial aneurysmal hemorrhages are rare and the incidence in children of less than 15 years is 0.05 to 0.09 per 100,000 person-years. ${ }^{1}$ Interestingly, in the pediatric population, the overall incidence of aneurysm is noted to be higher in males compared with females (3:2) as opposed to adults (1:2). ${ }^{1}$ In the case discussed here, the parent artery was occluded due to the distal location, size, and configuration of the aneurysm. This patient represents the first report of a noninfective, large peripheral artery aneurysm in a child in the published literature to our knowledge.

Distal segment middle cerebral artery (MCA) aneurysms account for only 1.1 to $1.7 \%$ of aneurysms. ${ }^{2}$ A literature review yielded nine cases of ruptured cortical MCA (M4 region) aneurysms, all in adult patients. ${ }^{2}$

The variation in the developmental anatomy of cerebral arterial vessels during embryogenesis can result in the formation of aneurysms, the first stage of which happens during angiogenesis. ${ }^{3}$ The intradural segments of the intracranial arteries have a decreased amount of elastic lamina and thinner adventitia, which increases the risk for aneurysm formation. 4

There are multiple intrinsic and environmental factors, including hemodynamic stress, congenital, infectious (15\%), and traumatic (5-10\%), which may affect structural changes of cerebral vessels resulting in the development of aneurysm in children. ${ }^{1,5}$

Surgery is considered the best option for the treatment of ruptured cortical aneurysms with intracerebral hemorrhage, since it allows both management of mass effect and aneurysmal exclusion. ${ }^{2}$

\section{Conclusion}

Ruptured cortical aneurysm should be considered as a rare etiology along with other common differentials such as arteriovenous malformation or tumors in pediatric patients with spontaneous intracerebral bleed.

\section{Conflict of Interest}

None declared.

\section{Acknowledgment}

The authors would like to thank Dr. Peter Jessup (pathologist) and Dr. T Tayiba (pathology registrar) Royal Hobart Hospital for their contribution to the paper by providing histopathology slides. Also, they thank Noelene Westerneng (Library assistant, Launceston General Hospital) for checking the references.

\section{References}

1 Sorteberg A, Dahlberg D. Intracranial non-traumatic aneurysms in children and adolescents. Curr Pediatr Rev 2013;9(4): 343-352

2 Ricci A, Di Vitantonio H, De Paulis D, et al. Cortical aneurysms of the middle cerebral artery: a review of the literature. Surg Neuro Int. 2017; 8:117.

3 Menshawi K, Mohr JP, Gutierrez J. A functional perspective on the embryology and anatomy of the cerebral blood supply. J Stroke 2015;17(2):144-158

4 Jung KH. New pathophysiological considerations on cerebral aneurysms. Neurointervention 2018;13(2):73-83

5 Krings T, Geibprasert S, terBrugge KG. Pathomechanisms and treatment of pediatric aneurysms. Childs Nerv Syst 2010; 26(10):1309-1318 